\title{
DESCRIPTION ET CONTRÔLE DE L'OVOGENÈSE GHEZ LES CRUSTACÉS SUPÉRIEURS
}

\author{
Hélène CHARNIAUX-COTTON \\ Laboratoire de Sexualité et Reproduction des Invertébrés, \\ Tour 32, Université de Paris VI, \\ 4, Place Jussieu, 75230 Paris Cédex 05 (France)
}

RÉSUMÉ

L'ovogenèse chez les Crustacés supérieurs se divise en deux étapes. La première va de l'ovogonie à la fin de la première croissance (prévitellogenèse); la seconde est la vitellogenèse. Cette dernière n'a lieu qu'en saison de reproduction alors que la première étape a lieu toute la vie de la femelle. Les ovogonies primaires sont enfermées dans une zone germinative constituée de cellules mésodermiques qui entourent complètement chaque cellule germinale. Elles sont caractérisées, chez Orchestia gammarella, par la présence de canalicules intranucléaires. Les mitoses ovogoniales n'ont lieu que dans la zone germinative. Des gonies quittent cette zone et entrent en prophase de méiose; les chromosomes s'effacent et la première croissance a lieu. Elle est caractérisée par une très grande abondance de ribosomes.

Durant la saison de reproduction, plusieurs cycles de vitellogenèse, concernant un nombre d'ovocytes variable avec les espèces, s'effectuent successivement. Durant chaque cycle, tous les gros ovocytes sont au même stade et les jeunes ovocytes restent bloqués en prévitellogenèse. La maturation, chez les Amphipodes, commence dans l'ovaire peu avant la mue ; les oufs sont pondus au stade diacinèse et la maturation s'achève avant la pénétration du spermatozoìde.

Il est connu que la vitellogenèse est sous le contrôle inhibiteur exercé par le complexe neurosécréteur, logé dans les pédoncules oculaires chez les Décapodes. Un signal bref positif de démarrage intervient certainement. En effet, chez les Crevettes épédonculées, comme chez les normales, durant la vitellogenèse, les jeunes ovocytes restent bloqués en fin de prévitellogenèse malgré la disparition de l'hormone inhibitrice. Il semble que l'exuviation constitue directement ou indirectement ce signal.

Les ovaires de Crustacés possèdent des enzymes intervenant dans la biosynthèse des hormones stéroïdes mais aucune action de ces dernières n'a pu être démontrée.

\section{I. - ÉTUDES DESCRIPTIVES DE L'OVOGENÈSE}

L'ovogenèse chez les Crustacés Malacostracés (supérieurs) a été rarement décrite de façon complète de l'ovogonie à la maturation de l'ovocyte et les mécanismes contrôlant ses diverses étapes sont encore peu connus. Nous avons fait nos 
recherches essentiellement sur 1'Amphipode Orchestia gammarella et la Crevette Lysmata seticaudata. Les observations faites chez quelques autres Malacostracés nous permettent de penser que les données obtenues ont une valeur assez générale.

\section{A. - Développement de l'appareil génital femelle}

A la fin de la vie larvaire ou à l'éclosion chez les espèces sans larve (comme O. gammarella) 1'appareil génital est identique dans les deux sexes. Il est constitué par deux cordons de cellules mésodermiques enfermant des gonies et entièrement compris dans le thorax (fig. I). Ces cordons sont accolés au septum péricardial

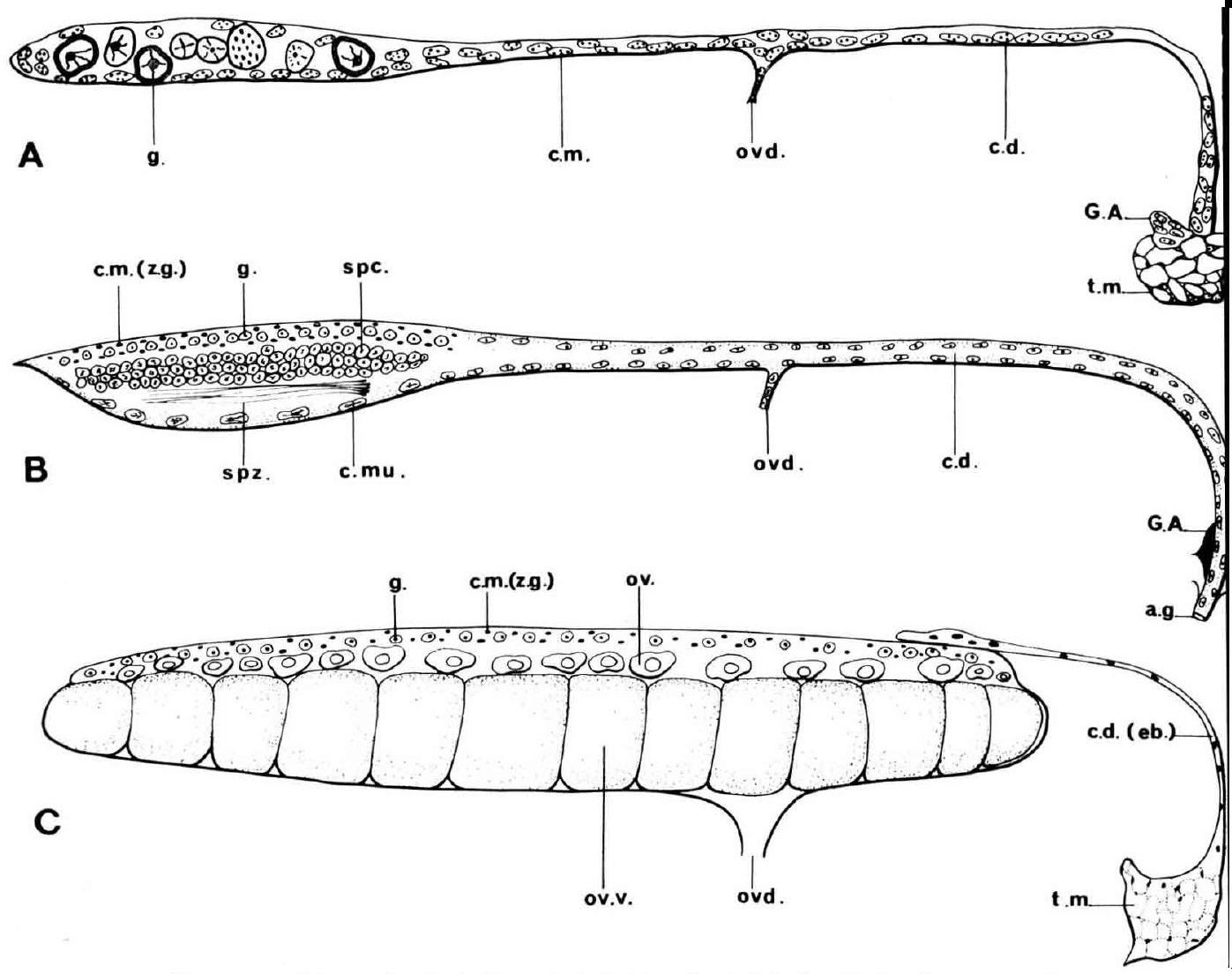

FIG. I. - Schéma du développement de l'appareil génital chez Orchestia gammarella

A : stade indifférencié ( $\mathrm{I}^{\text {re }}$ intermue); la région antérieure est peuplée de gonies (g.); présence des ébauches des voies déférentes des deux sexes (ovd., c.d.) et des ébauches des glandes androgènes (G. A.).

$B$ : jeune mâle, peu après la mue de différenciation externe du sexe ( $5^{e}$ mue). L'ébauche de l'oviducte (ovd.) disparaît plus tard.

$\mathrm{C}$ : femelle en vitellogenèse; l'ébauche de la G. A. ne se distingue plus dans le tissu mésenchymateux (t.m.).

a.g. : apophyse génitale ; c.d. : canal déférent ; c.d. (eb.) : ébauche du canal déférent persistant chez la femelle ; c.m. : cellule mésodermique; c.m. (Z.g.) : cellules mésodermiques formant le stroma de la zone germinative qui enferme les gonies ; c.mu. : cellules bordant la cavité testiculaire ; g. : gonie ; G. A. : glande androgène; ovd. : oviducte; ov.v. : ovocyte en vitellogenèse ; spc. : spermatocyte; spz. : spermatozoïde; t.m. : tissu mésenchymateux.

(Charniaux-Cotton, I965). 
de part et d'autre du tube digestif. Les ébauches des voies déférentes des deux sexes sont présentes. Très rapidement, dès le deuxième intermue chez $O$. gammarella (CHARNIAUX-CotTon, I959), vers le troisième intermue chez les Écrevisses et les Crabes (PAYEN, données non publiées), certaines gonies entrent en prophase de méiose et forment des ovocytes disséminés tout le long du cordon ovarien. Soulignons

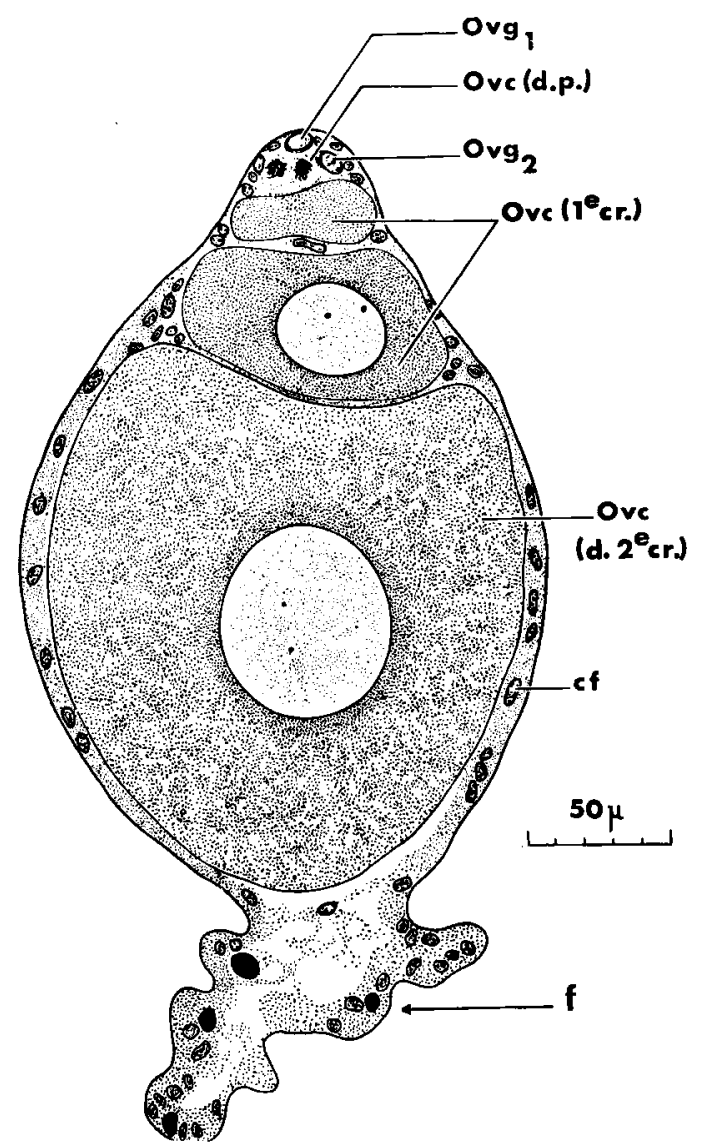

FIG. 2. - Coupe transversale de l'ovaire d'Orchestia gammarella en début de vitellogenèse

c.f. : cellule folliculeuse ; f. : follicule d'un ovocyte récemment pondu ; Ovg.1 : ovogonie primaire (dans la zone germinative); Ovg.2: Ovogonie secondaire (sortie de la zone germinative); Ovc. (d.p.) : ovocyte en début de prophase de méiose ; Ovc. ( ${ }^{\text {re }} \mathrm{cr}$.) : ovocyte en première croissance (prévitellogenèse) ; Ovc. (d. $2^{\mathrm{e}} \mathrm{cr}$.) : ovocyte en deuxième croissance (vitellogenèse). (Meusy, 1972).

que 1'ovogenèse démarre plus tôt que la spermatogenèse chez $O$. gammarella et chez l'Écrevisse. L'ovaire acquiert rapidement sa structure définitive. C'est un organe tubulaire dont les coupes transversales sont identiques d'une extrémité à l'autre. Il ne comporte que deux catégories cellulaires : des cellules germinales et des cellules mésodermiques ; il est entouré d'une lame basale (fig. 2). 


\section{B. - La zone germinative}

Chez O. gammarella, des ovogonies, que nous appelons primaires, sont enfermées dans un tissu de cellules mésodermiques. L'ensemble forme une étroite bande longitudinale accolée à la lame basale; je l'ai nommée : zone germinative. Cette zone germinative persiste toute la vie de la femelle. Tant que les gonies sont enfermées dans ses cellules mésodermiques, elles n'entrent pas en gamétogenèse. Elles y effectuent des mitoses en toutes saisons.

Les ovogonies primaires ont des dimensions de $9 \times$ II, $5 \mu$ avec un noyau de $8 \times$ ro $\mu$. L'étude en microscopie électronique (MEUSY, I968) montre que chaque gonie est complètement entourée par des cellules mésodermiques et est ainsi séparée des autres et de la lame basale (fig. 3) ; ses échanges avec l'hémolymphe passent donc obligatoirement par le tissu mésodermique.

Le cytoplasme des ovogonies comporte beaucoup de ribosomes libres ou groupés en polyribosomes. Le réticulum endoplasmique est très pauvre et constitué de petites vésicules ovoïdes, lisses ou peu granulaires. L,es mitochondries possèdent des crêtes transversales. Aucune polarité n'a été notée dans leur répartition. Les dictyosomes ne sont ni abondants ni volumineux.

Les noyaux présentent une particularité que nous n'avons pu interpréter : la membrane interne de l'enveloppe nucléaire donne naissance à de fins canalicules aplatis, d'environ $200 \AA$ d'épaisseur, qui s'enfoncent profondément au sein du noyau. Ils comportent des pores semblables à ceux de l'enveloppe nucléaire. Ils atteignent parfois le nucléole ; en face de leur ouverture, dans le cytoplasme, existe un nuage opaque aux électrons qui rappelle la substance nucléolaire (fig. 4).

Les cellules mésodermiques de la zone germinative ont un réticulum endoplasmique formé de petites vésicules lisses ou peu granulaires, des mitochondries de petite taille, à crêtes transversales et des dictyosomes rares. Des vacuoles de gros diamètre $(5 \mu)$ sont peut-être des lysosomes. Les noyaux sont allongés $(7 \times 2,5 \mu)$ et montrent une chromatine dense.

Il nous apparaît très important de souligner que la zone germinative de l'ovaire diffère de celle du testicule uniquement par un nombre plus faible de gonies; en particulier, l'ultrastructure des ovogonies et celle des spermatogonies sont identiques.

Une zone germinative de structure similaire à celle d'O. gammarella et également accolée à la lame basale a été observée chez les divers Décapodes étudiés : Lysmata seticaudata, Pandalus borealis (Charniaux-Cotron, I96r, I965), le Crabe Carcinus maenas, le Pagure Clibanarius misanthropus, l'Araignée de mer Maia squinado (MEusy, I972). L'étude en microsciope électronique réalisée chez les trois dernières espèces n'a pas montré de canalicules intranucléaires dans les gonies primaires. Ceci est peut-être à rapprocher de l'impossibilité d'obtenir la spermatogenèse chez les femelles de ces espèces par greffes de glandes androgènes alors que l'inversion des ovaires en testicules peut être induite toute la vie de la femelle d'O. gammarella (voir Charniaux-Cotron, I965, I972). C'est en effet dans la zone germinative que les gonies se différencient en spermatogonies ou ovogonies selon qu'elles ont reçu ou non de l'hormone androgène comme l'ont prouvé les études expérimentales. 
Il est intéressant de rapprocher la zone germinative des Crustacés de l'ébauche génitale des Vertébrés laquelle est également constituée par des gonocytes entourés de cellules mésodermiques (Franchi et MANDL, I964).

\section{C. - De l'ovogonie secondaire à la fin de la première croissance}

Des ovogonies sortent de la zone germinative par un mécanisme non élucidé et deviennent alors des ovogonies secondaires. Nous avons observé que cette sortie s'effectue toute l'année chez $O$. gammarella, chez $L$. seticaudata, donc en période de non reproduction comme en période de pontes. Chez Gecarcinus lateralis, WEITzMAN (I966) observe des " divisions ovogoniales " toute la vie du Crabe. Nous n'avons jamais observé de mitoses parmi les ovogonies secondaires d'O. gammarella et de L. seticaudata.

Chez $O$. gammarella, les ovogonies secondaires ne présentent plus, au microscope électronique, les invaginations de la membrane nucléaire interne. Leur cytoplasme, contrairement à celui des gonies primaires, se colore en rose par la méthode de Unna-Pappenheim, ce qui prouve qu'elles s'enrichissent en ARN (MEUSY, I972). Sur une coupe transversale, une ovogonie secondaire voisine avec un à trois ovocytes, aux divers stades de la prophase méiotique ; ceux au stade pachytène ont un diamètre de I9 $\mu$ et un noyau de I4 $\mu$ (fig. 2 ). Sous ces cellules sont superposés, en taille croissante, 2 à 3 ovocytes dont la chromatine n'est plus visible qu'à l'état de trace. Cette disparition se fait après le stade diacinèse. Le nucléole qui avait disparu au stade pachytène, est très développé. Après l'extension des chromosomes, l'ovocyte va croître d'une façon importante jusqu'à atteindre $200 \mu$ environ dans sa plus grande dimension avec une vésicule germinative de $60 \mu$ de diamètre. De plus, il s'entoure d'un follicule simple à noyaux espacés. Ces follicules apparaissent formés à partir des cellules mésodermiques formant une assise cellulaire à la périphérie de l'ovaire. Ces cellules sont probablement dérivées de la zone germinative. Durant cette première croissance, le cytoplasme reste fortement basophile et pyroninophile. Il apparaît bourré de ribosomes libres et pauvre en réticulum endoplasmique (ZERBIB, donnée non publiée). Des lipides commencent à s'accumuler, surtout à la périphérie de l'ovocyte.

La première étape de l'ovogenèse qui se termine à la fin de la première croissance a lieu toute l'année chez L. seticaudata (CharnIaux-CotTon et TourR, I973) et chez $O$. gammarella. Nous 1'appelerons : prévitellogenèse.

\section{D. - La vitellogenèse}

La vitellogenèse est définie comme 1'accumulation par l'ovocyte d'enclaves lipidiques et protéiques appelées à être utilisées par l'embryon. Chez les Crustacés, elle se distingue aisément de la prévitellogenèse par le fait qu'elle n'a lieu qu'à partir d'un certain âge et à certaines saisons alors que la prévitellogenèse est continue : généralement, plusieurs cycles de vitellogenèse se succèdent.

$L_{a}$ vitellogenèse provoque un accroissement très important : ainsi chez $O$. gammarella, les ovocytes vont passer d'un diamètre de $200 \mu$ à un diamètre de $800 \mu$ avec une vésicule germinative de $60 \mu$. Les ovocytes en vitellogenèse sont plus ou moins nombreux selon les espèces (I2 par ovaires chez $O$. gammarella). Ils sont tous au même stade et il n'y a pas d'ovocytes de taille intermédiaire entre eux et 
les ovocytes arrivés en fin de prévitellogenèse. Donc aucun de ces derniers n'entre en vitellogenèse pendant la durée de ce processus.

L'entrée en vitellogenèse semble caractérisée par le développement du réticulum endoplasmique qui se forme par bourgeonnement de la membrane externe de l'enveloppe nucléaire, comme le montrent les études réalisées chez l'Araignée de mer Libinia marginata (HINSCH et CONE, I969) et chez O. gammarella. Un matériel dense aux électrons devient visible dans les citernes. Une caroténolipoprotéine qui n'existe que dans l'hémolymphe des femelles ou " protéine femelle " concoure également à la formation du vitellus en pénétrant dans l'ovocyte par micropinocytose (citons Frentz, rg6o; Meusy, Charniaux-Cot'Ton et Croisil. 1972). La synthèse de cette protéine est contrôlée par l'ovaire (MEUSY, JuNERA, CROISILLE, I97I). L,es plaquettes vitellines, d'abord visibles à la périphérie, emplissent peu à peu tout le cytoplasme.

Durant la vitellogenèse, le follicule montre des noyaux de plus en plus nombreux et serrés. Son rôle n'a pas été étudié ; peut-être est-il limité à la formation de l'enveloppe vitelline comme cela semble être le cas chez les Insectes.

\section{E. - La maturation}

Chez $O$. gammarella, la vitellogenèse s'effectue dans l'intervalle de temps qui sépare deux mues (23 jours). Peu avant l'exuviation (stade $\mathrm{D}_{2}$ de l'intermue), la vésicule germinative migre à la périphérie de l'ovocyte. Après l'exuviation et avant la ponte, l'enveloppe de la vésicule germinative disparaît. Les chromosomes sont visibles au bord de l'ovocyte (fig. 5). Ils baignent dans le nucléoplasme homogène qui a pris un aspect étoilé. Les tétrades (au nombre de 24) présentent un aspect d'haltères. Les ovocytes sont pondus au stade diacinèse. Une demi-heure après la ponte, la métaphase de première division est bien visible : le fuseau est perpendiculaire à la surface de l'ovocyte. Une heure après la ponte, le premier globule polaire est expulsé et se loge sous l'enveloppe (chorion) de l'œuf. L'intercinèse est de courte durée ; il n'y a pas reconstitution de la membrane nucléaire. Le fuseau de deuxième division est également perpendiculaire à la surface de l'œuf ; il est plus étroit que celui de première division. Le deuxième globule polaire est émis et deux heures après la ponte les phénomènes de maturation sont terminés.

La pénétration du spermatozoïde a lieu à la fin de ces phénomènes de maturation. Précisons que les spermatozoïdes sont projetés sur la face ventrale de la femelle lors de l'appariement qui provoque la ponte. Les spermatozoïdes sont donc présents lorsque les œufs sont pondus mais ceux-ci sont enclos dans un sac muqueux que les spermatozoïdes traversent environ I h 30 après la ponte (Charniaux-Cotron et SaINT-PaLAIS, données non publiées).

Chez le Gammare, Gammarus duebeni, les phénomènes sont comparables : la maturation débute entre la mue et la ponte et se termine après celle-ci ; elle est suivie de la fécondation (LEROUx, I933). Chez le Pagure Diogenes pugilator (BLOCH, I935) l'émission du premier globule polaire aurait lieu après la pénétration du spermatozoïde.

Comme on le voit, la maturation de l'œuf des Crustacés supérieurs a été peu étudiée. 


\section{II. - CONTRÔLE DES PHÉNOMÈNES DE L'OVOGENÈSE}

\section{A. - La prévitellogenèse}

La prévitellogenèse étant continue, elle ne nécessite probablement aucun contrôle pour s'effectuer. Rappelons que nous avons pu démontrer qu'elle est l'évolution spontanée des gonies qui n'ont pas subi l'action de l'hormone androgène; c'est ce que j'ai appelé l'“ autodifférenciation ovarienne ". Ceci explique que la prévitellogenèse se rencontre assez souvent dans les testicules de Malacostracés, des gonies ayant pu échapper à l'hormone mâle; les ovocytes évoluent normalement parmi les spermatocytes et ils acquièrent un follicule. Cette constatation ainsi que d'autres faits (CHARNIAUx-CoTron, I96I) permettent de penser que les cellules germinales agissent sur les cellules mésodermiques et provoquent la constitution du follicule. De plus, les cellules folliculeuses se transforment en cellules phagocytaires lorsque les ovocytes ne sont pas pondus.

\section{B. - Contrôle endocrine de la vitellogenèse}

Il est connu, depuis les expériences d'ablation des pédoncules oculaires réalisées par Panouse (I943) chez la Crevette Palaemon serratus, que le complexe neurosécréteur cérébral, organe X-glande du sinus (localisé dans les pédoncules oculaires), contrôle la vitellogenèse par effet inhibiteur (voir DEMEUSY, I970). L'existence d'une hormone positive de vitellogenèse n'a pas été démontrée.

Etant donné qu'aucun nouvel ovocyte n'entre en vitellogenèse pendant que celle-ci se déroule, on pouvait penser que l'inhibition cérébrale ne cesse qu'un court moment pour en permettre le démarrage (fig. 6). Or, nous avons observé que les ovaires des Crevettes Lysmata seticaudata privées du complexe neurosécréteur par pédonculectomie présentent les mêmes phénomènes que les ovaires des individus normaux (fig. 7). Il doit donc exister un signal bref pour le déclenchement de la vitellogenèse. Diverses données permettent de penser que ce signal est la mue (exuviation) ou l'état endocrine corrélatif c'est-à-dire un taux minimal d'hormone de mue (ADELUNG, I97I) :

- chez diverses espèces comme Lysmata, 0 . gammarella, une vitellogenèse débute peu après chaque exuviation ;

- après l'ablation, en hiver (période de repos génital), des pédoncules oculaires de Lysmata, la vitellogenèse ne démarre qu'après la première mue post-opératoire (Charniaux-CotTon et Touir, I973) ;

- chez $O$. gammarella, 1'injection d'ecdystérone peu avant l'exuviation retarde cette dernière et également le démarrage d'une nouvelle vitellogenèse (BLANCHE'T, I972) ;

- chez le Crabe Carcinus maenas, 1'extirpation des glandes de mue est toujours suivie de vitellogenèse (DEMEUSY, I970).

Donc la vitellogenèse apparaît contrôlée de la façon suivante : les facteurs externes provoquent la levée de l'inhibition cérébrale; le taux minimal d'ecdysté- 
rone provoque le démarrage de la vitellogenèse. I1 y a tout lieu de penser que cette régulation agit sur l'admission de la protéine femelle par micropinocytose dans les ovocytes.

\section{C. - Contrôle de la maturation}

Comment sont contrôlées la fin de la vitellogenèse et la maturation? Chez les espèces comme $L$. seticaudata, $O$. gammarella où la vitellogenèse s'effectue exactement en un intermue, les phénomènes de la fin de l'intermue jouent probablement un rôle régulateur mais les preuves expérimentales manquent. Les phénomènes sont probablement complexes puisque l'ovaire en vitellogenèse exerce un contrôle sur le déroulement de 1'intermue ; en effet, si l'hormone de mue (ecdystérone) est injectée dans une femelle $O$. gammarella en vitellogenèse au début de l'intermue, les phénomènes préparatoires à l'exuviation sont déclenchés mais ensuite bloqués de façon telle que 1'intermue et donc la vitellogenèse ont une durée normale (23 jours) ( $B \mathrm{I}_{\text {.AN- }}$ ChET et Charniaux-Cotton, I97I). Nous avons recherché une action éventuelle de la I-méthyladénine sur la reprise de la méiose dans les ovocytes d'O. gammarella; cette substance isolée par KANATANI et al. (r969) chez 1'Étoile de mer est responsable de la maturation. L'injection dans une femelle ou l'incubation de l'ovaire dans une solution n'a montré aucune action, quel que soit le stade de vitellogenèse. Les doses utilisées étaient sensiblement plus fortes que celles de KanatanI (environ ro $\mu \mathrm{g}$ par $\mathrm{ml}$ d'eau de mer ou par g d'O. gammarella).

\section{D. - Présence d'hormones stéroïdes dans les gonades}

Les Crustacés ne peuvent synthétiser le cholestérol à partir de l'acétate ou du mévalonate. En revanche, après injection de cholestérol-14C dans la Langouste Panulirus japonicus, les hormones stéroïdes radioactives suivantes ont été identiffées dans les ovaires : progestérone, I $7 \alpha$-hydroxyprogestérone, androstènedione, testostérone ; 1'hépatopancréas contient en outre de la déoxycortiscotérone et de la corticostérone (KanAzawa et Teshima, I97I). Ces expériences ne prouvant pas le rôle des ovaires dans ce métabolisme, leurs auteurs (TEshrma et Kanazawa, I97 I $a$ ) ont réalisé des incubations d'ovaire du Crabe Portunus trituberculatus en présence de progestérone- $4^{-{ }^{14} \mathrm{C}}$; ils ont constaté la formation de $\mathrm{I} 7 \alpha$-hydroxyprogestérone, testostérone et déoxycorticostérone. L'absence d'androstènedione s'explique probablement par sa rapide transformation en testostérone. En effet chez Astacus astacus les ovaires se montrent capables de réduire 1'androstènedione en testostérone (BJöRKHEM et DANIELSSON, I97I).

Les testicules des Crustacés contiennent également des enzymes intervenant dans le métabolisme des stéroïdes. Ceux de Portunus trituberculatus incubés avec de la progestérone-4-4 ${ }^{14} \mathrm{C}$ métabolisent celle-ci en $\mathrm{I} 7 \alpha$-hydroxyprogestérone et testostérone ('TESHIMA et KaNAZAWA, I97 I $b$ ). La même remarque que pour l'ovaire peut être faite en ce qui concerne l'absence d'androstènedione puisque tout l'appareil génital mâle du Homard Homarus americanus (GILGAN et IDLER, I967) et celui du Crabe Carcinus maenas (BLANCHET, OzON et MEUSY, 1972) présentent une forte activité de la $\mathrm{I}_{7} \beta$-hydroxystéroïde oxydo-réductase.

La différence entre ovaires et testicules pourrait être l'incapacité de ces derniers à transformer la progestérone en désoxycorticostérone. Cependant, chez les Crabes 
Callinectes sapidus (TCHOLAKIAN et EIK-Nes, I969) et Carcinus maenas (BLANCHET et al., I972) une 2I-hydroxylase existe dans le canal déférent et les glandes androgènes; malheureusement les testicules n'ont pas été étudiés.

Enfin la conversion de la testostérone en œestrogènes n'a pas été obtenue chez les Crustacés.

L'injection de diverses hormones stéroïdes chez des mâles et des femelles d'O. gammarella ne nous a pas permis de mettre en évidence une action quelconque de ces hormones.

\section{SUMMARY}

\section{DESCRIPTION AND CONTROL, OF OOGENESIS \\ IN MAI,ACOSTRACAN CRUSTACEA}

Oogenesis in malacostracan crustacea may be divided into two stages. The first stage begins with ovogonia and goes up to the end of first growth or previtellogenesis; the second stage is vitellogenesis. It only occurs in the reproduction season, while the first stage continues all during the life of the female.

The primary oogonia are enclosed in a germinative zone constituted by mesodermic cells which completely surround each germinative cell. They are characterized in Orchestia gammarella by the presence of intranuclear canaliculi. Oogonial mitoses take place only in the germinative zone. Gonia leave this zone and carry out meiosis prophase, the chromosomes disappear and first growth begins, which is characterized by a very great abundance of ribosomes.

During the reproduction period, several vitellogenesis cycles occur successively in which the number of oocytes involved depends on the species. During each cycle, all the big oocytes are in the same stage and the young oocytes stay in previtellogenesis. Maturation in amphipoda begins in the ovary shortly before the molt; eggs are laid in the diacinese stage and maturation is completed before fertilization.

It is known that the neurosecretory system, located in the eyestalks of decapoda, exercises an inhibitory control on vitellogenesis. A short positive go signal certainly intervenes. In fact, during vitellogenesis in eyestalkless prawns, as in normals, the young oocytes stay growth-stopped in spite of the disappearance of the inhibitory hormone. Ecdysis seems directly or indirectly to constitute this signal.

The ovaries of crustaceans possess enzymes involved in steroid hormone biosynthesis, but no action of these hormones has been demonstrated.

\section{RÉFÉRENCESS BIBLIOGRAPHIQUES}

Adelung D., I971. Untersuchungen zur Häutungsphysiologie der dekapoden krebse am Beispiel der Strand krabbe Carcinus maenas. Helgoländer viss. Meeresunters., 22, 66-I 19.

BJörkhem I., Danielsson H., I97I. Metabolism of 3-oxosteroids in crayfish tissues. Comp. Biochem. Physiol., $38 b, 315-326$.

Blanchet M.-F., I972. Effets sur la mue et sur la vitellogenèse de la $\beta$-ecdysone introduite aux étapes $A$ et $\mathrm{D}_{2}$ du cycle d'intermue chez Orchestia gammarella Pallas (Crustacé, Amphipode). Comparaison avec les effets de la $\beta$ - et de l' $\alpha$-ecdysone aux autres étapes de l'intermue. C. $R$. Acad. Sci., 274, 30153018.

Blancher M.-F., Charniaux-Cotton H., r97x. Contrôle du déclenchement et de la durée de la période D du cycle d'intermue par l'ecdystérone, chez le Crustacé Amphipode Orchestia gammarella (PAllas); interaction avec la vitellogenèse. C. R. Acad. Sci. Paris., 272, 307-3Io.

Blanchet M.-F., Ozon R., Meusy J. J., I972. Metabolism of steroids, in vitro in the male Crab Carcinus maenas linet. Comp. Biochem. Physiol., $41 \mathrm{~B}, 25 \mathrm{I}-26 \mathrm{I}$.

Block F., I935-37. Contribution à l'étude des gamètes et de la fécondation chez les Crustacés Décapodes. Trav. Soc. Zool. Wimereux, 12, 185-279.

Charniaux-Cotron H., I959. Étude comparée du développement postembryonnaire de l'appareil génital et de la glande androgène chez Orchestia gammarella et Orchestia mediterranea (Crustacé, Amphipode) Autodifférenciation ovarienne. Bull. Soc. Zool., 84, 105-115. 
Charniaux-Cotron H., 196r. Physiologie de la gonade de Lysmata seticaudata (Crevette protérandrique) chez les individus normaux et les femelles masculinisées. C. R. Acad. Sci., 252, r99-20r.

Charniaux-Cotton $H_{\text {., }}$ ig65. Hormonal control of sex differentiation in Invertebrates. In Organogenesis, De Haan, R. und Ursprung, H. ed. Holt, Rinehart and Winston, N. Y., 7or-74o.

Charniaux-Cotton H., I972. Recherches récentes sur la différenciation sexuelle et l'activité génitale chez divers Crustacés supérieurs. In Cours et documents de Biologie, 3, édité par Gordon et Breach, I27-I78.

Charniaux-Cotion H., Touir A., I973. Contrôle de la prévitellogenèse et de la vitellogenèse chez la Crevette hermaphrodite Lysmata seticaudata RIsso. C.R. Acad. Sci., Séance du 9 Avril.

Demeusy N., I97o. Chez les Décapodes Brachyoures gonochoriques, quelques aspects de la sexualité. Bull. Soc. Zool. France, 95, 595-612.

Franchi L. L., Mande A. M., I964. The ultrastructure of germ cells in fetal and neonatal male rats. J. Embryol. exp. Morph., 12, 289-308.

Frentz R., 1960. Contribution à l'étude biochimique du milieu intérieur de Carcinus maenas linNé. Bull. Soc. Sci., Nancy, nouvelle série, 19, mém. I, I-r 76.

Gilgan N. W., IDLER D. R., 1967. The conversion of androstenedione to testoste by some Lobster (Homarus americanus MILNe EDWARDs) tissues. Gen. Comp. Endocrinol., 9, 319-324.

Hinsch G. W., Cone M. V., r969. Ultrastructural observations of vitellogenesis in the spider Crab Libinia emarginata L. J. Cell Biol., 40, 336-342.

Kanatani H., Shirai H., Nakanishi K., Kurokawa T., I969. Isolation and identification of meiosis inducing substance in starfish, Asterias amurensis. Nature, 221, 273-274.

Kanazawa A., Teshima S. I., 1971. In vivo conversion of cholesterol to steroid hormones in the spiny Lobster Pamulirus japonica. Bull. jap. Soc. Sci. Fish., 37 (9), 89r-898.

Leroux M. L., I933. Recherches sur la sexualité des gammariens. Suppl. Bull. Biol. France, Belgique, 16, 24-46.

Meusy J.-J., Ig68. Ultrastructure de la zone germinative et des gonies du testicule et de l'ovaire d'O.' gammarella P. (Crustacé, Amphipode). Ann. Sci. Nat., 10, Ior-1 6.

Meusy J.-J., I972. La gamétogenèse et la fraction protéique de l'hémolymphe spécifique du sexe femelle chez quelques Crustacés supérieurs : étude descriptive et rôle des glandes androgènes. Thèse, Université Paris, VI, no AO 6583 .

Meusy J.-J., Charniaux-Cotton H., Croisille Y., 1969. Étude par électrophorèse chez Orchestia gammarella (PALLAs) et Orchestia mediterranea costa (Crustacés, Amphipodes) des protéines de l'hémolymphe : comparaison entre les mâles, les femelles et les intersexués. C. R. Acad. Sci., 269, $74 \mathrm{I}-743$.

Meusy J.-J., Junera H., Croisille Y., 197I. Recherche de la "fraction rpotéique femelle " chez les Crustacés Amphipodes Orchestia gammarella ayant subi une inversion expérimentale du sexe. C. $R$. Acad. Sci., 273, 592-594.

Panouse J. B., I943. Influence de l'ablation du pédoncule oculaire sur la croissance de l'ovaire chez la Crevette Leander serratus. C. R. Acad. Sci., 217, 553-555.

TcholakiAN R. K., EIK-Nes K. B., I969. Conversion of progesterone to II-deoxycorticosterone by the androgenic gland of the blue Crab (Callinectes sapidus RATHBUN). Gen. Comp. Endocrinol., 12, Iクエ-I73.

Teshima S. I., Kanazawa A., I971 $a$. Bioconversion of progesterone by the ovaries of Crab, Portunus tribuberculatus. Gen. Comp. Endocrinol., 17, 152-157.

Teshima S. I., Kanazawa A., I97I $b$. In vitro bioconversion of progesterone to I7 $\alpha$-hydroxyprogesterone and testosterone by the sliced testes of Crab, Poriunus trituberculatus. Bull. Jap. Soc. Sci. Fish, 37 (6), 524-528.

Weitzman M. C., Ig66. Oogenesis in the tropical land Crab, Gecarcinus lateralis (Fréminville). $Z$. Zellforsch, 75, xog-119.

\section{PLANCHE I}

FIG. 3

Coupe transversale de la zone germinative de l'ovaire d'Orchestia gammarella. L'ovogonie primaire $\left(\mathrm{Ovg}_{2}\right)$ est complètement entourée par des cellules mésodermiques.

C.b. : corps basophile; L.b. : lame basale; n.m. : noyau de cellule mésodermique; $\mathrm{Ovg}_{1}$ : ovogonie primaire; Ovc : ovocyte ; t.m. : tissu mésodermique (MEUSY, I968). 
PLANCHE I

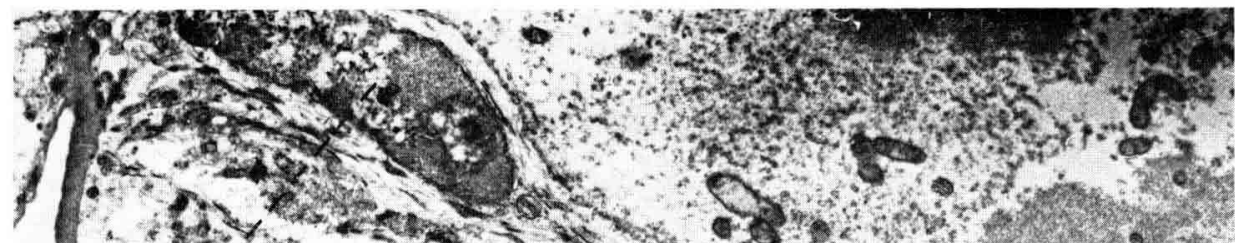

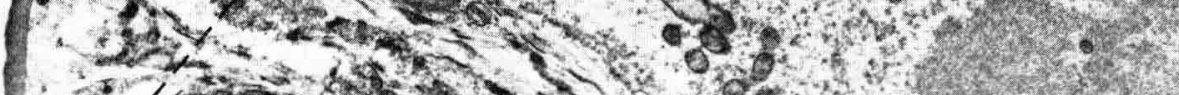

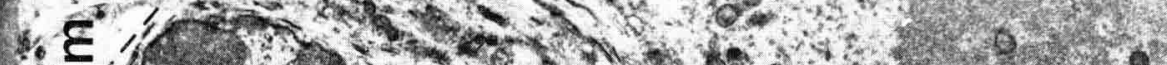

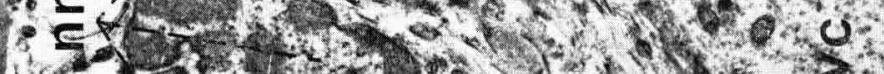

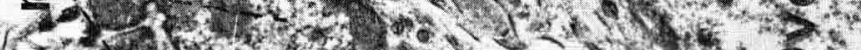

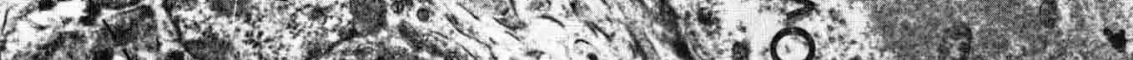

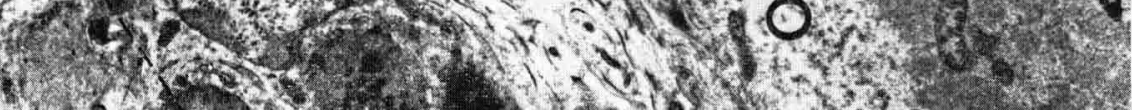

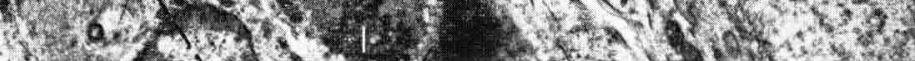

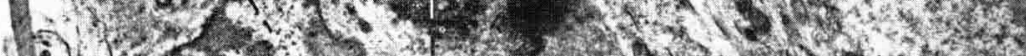

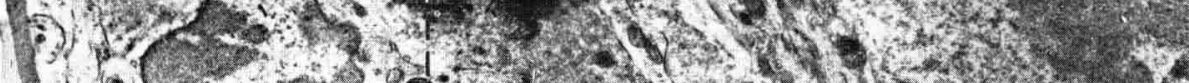

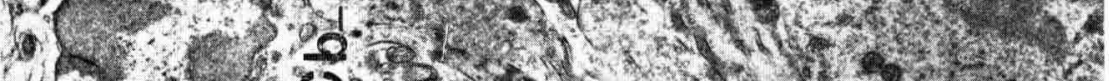

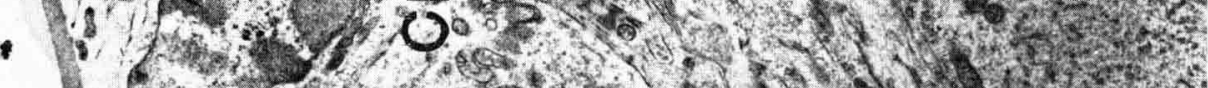

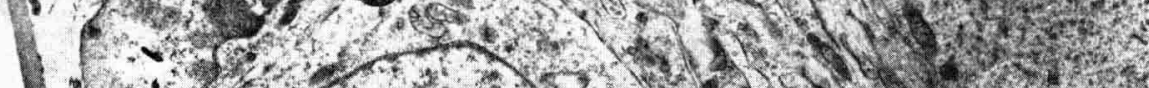

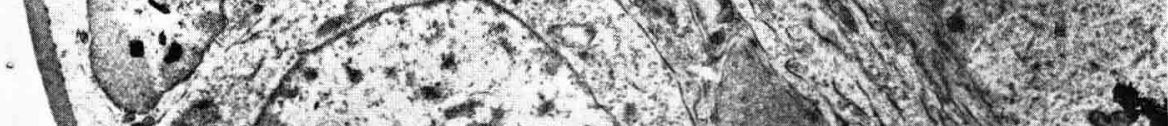

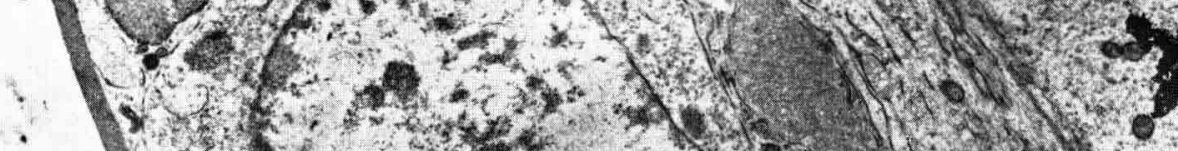

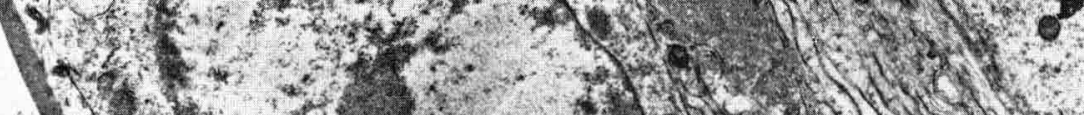
(2)

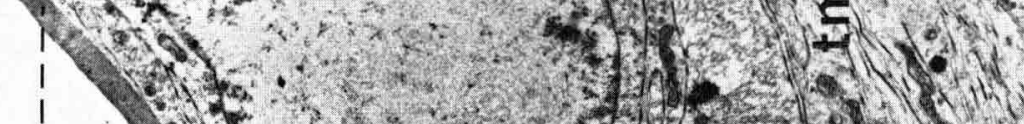
-

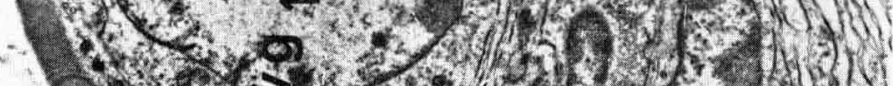
-

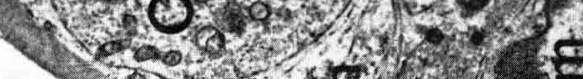

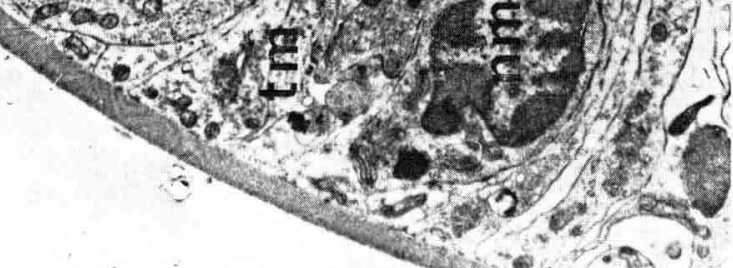




\section{PLANCHE II}

\section{Fig. 4}

Coupes d'ovogonie primaire mettant en évidence les canalicules intranucléaires.

A : Le canalicule intranucléaire (Ci) paraît atteindre le nucléole ; les pores sont bien visibles.

B : La continuité entre le canalicule intranucléaire et la membrane nucléaire interne est bien visible.

Cc : corps chromatoïde dans le cytoplasme face à l'ouverture d'un Ci. - Ci : canalicule intranucléaire. - Cy : cytoplasme. - $\mathrm{m}$ : mitochondrie. - mni : membrane nucléaire interne. $\mathrm{N}$ : noyau. - Nu : nucléole. - $\mathrm{p}:$ pore du canalicule intranucléaire. - pn : pore nucléaire (MEUSY, 1968). 


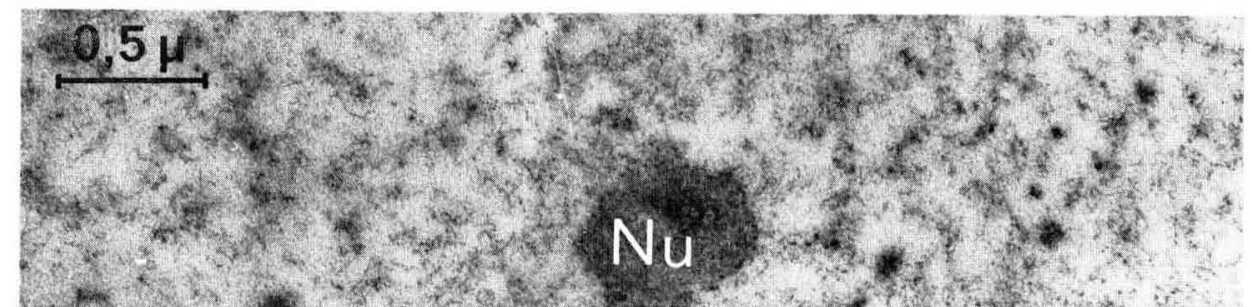

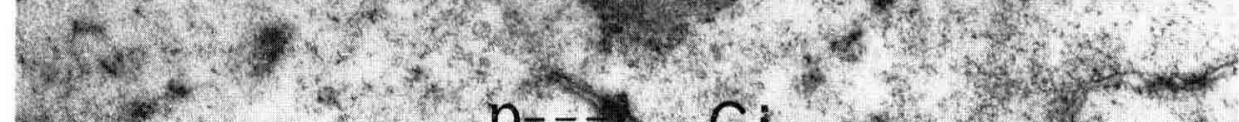

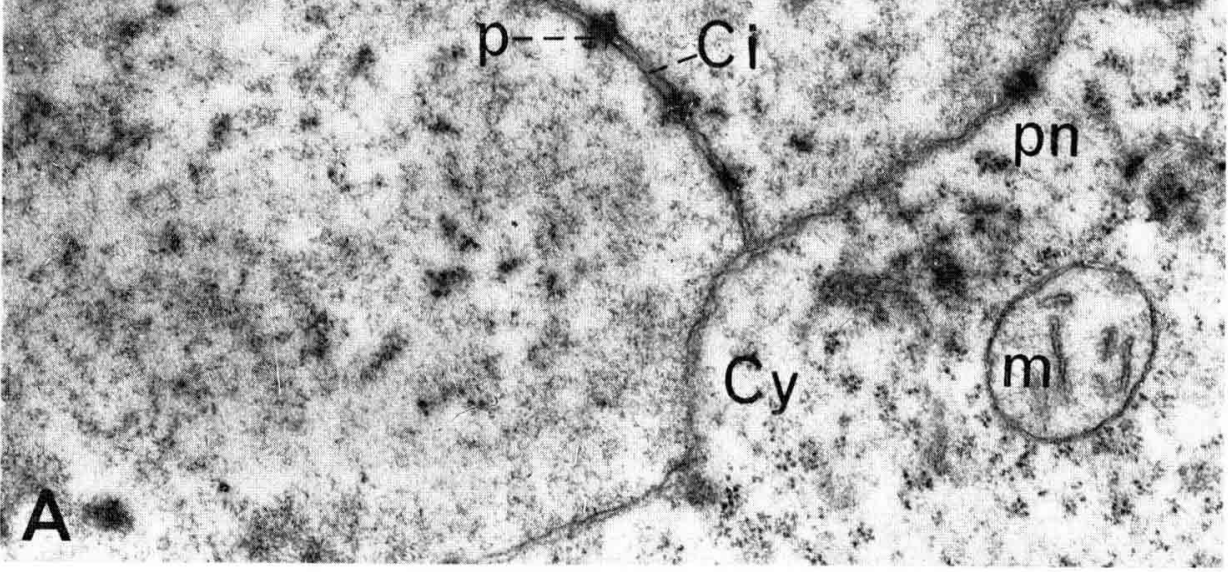

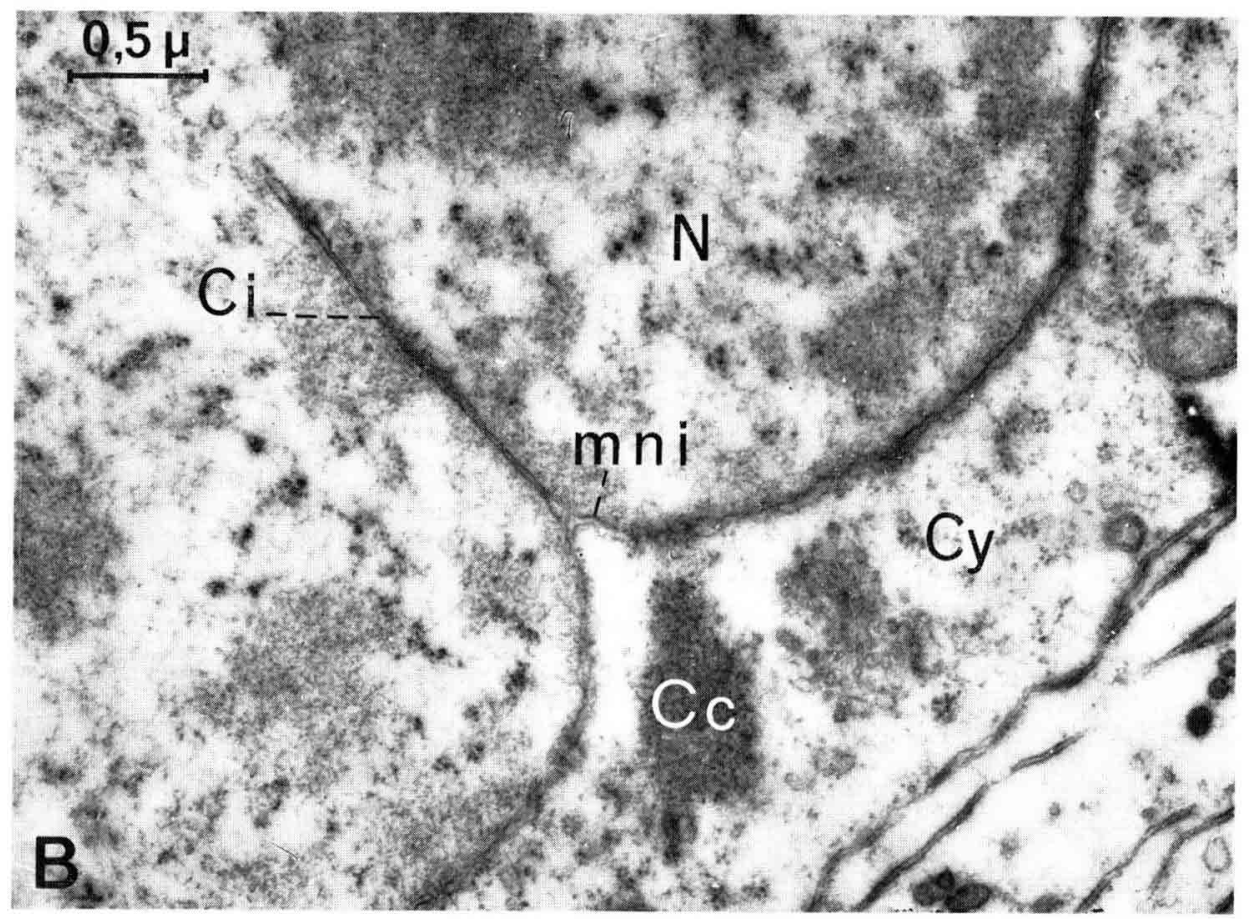




\section{PLANCHE III}

FIG. 5

Maturation de l'ovocyte d'Orchestia gammarella étudiée sur coupes après coloration par la réaction nucléale de Feulgen.

1. Ovocyte peu avant la ponte ou à la ponte : la vésicule germinative a migré à la périphérie de l'ovocyte avant l'exuviation ; après cette dernière, la membrane nucléaire a disparu et les chromosomes sont maintenant visibles, au stade diacinèse.

2. Une demi-heure après la ponte : métaphase de première division.

3. Trois quarts d'heure après la ponte : fin d'anaphase de première division.

4. Une heure un quart après la ponte : formation du premier globule polaire.

5 et 6 . Une heure et demie après la ponte : prophase et métaphase de deuxième division. 

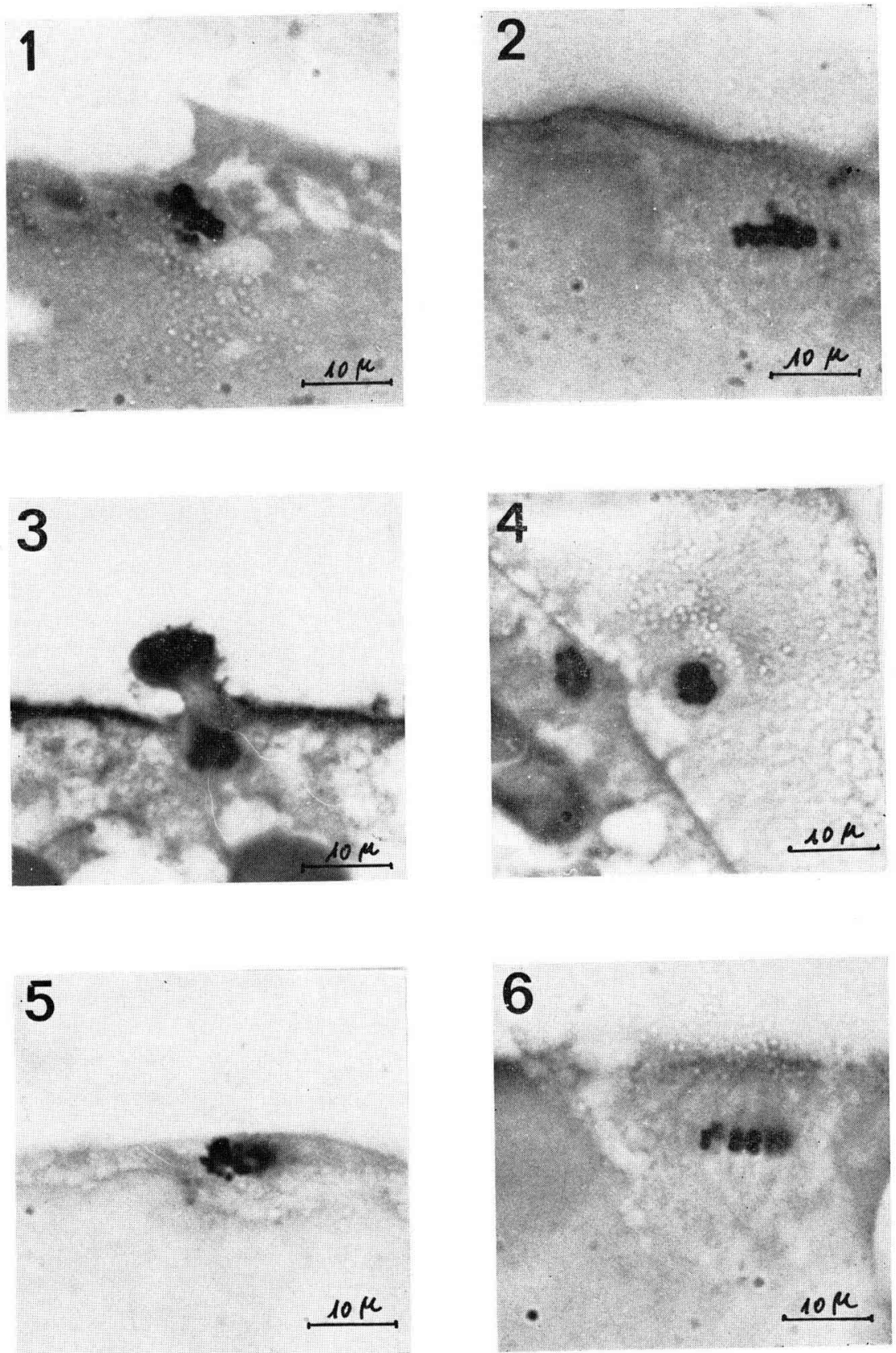

H. CHARNIAUX-COTTON 


\section{PLANCHE IV}

FIG. 6

Coupe d'ovaire chez la crevette Lysmata seticaudata en période de reproduction. Tous les ovocytes en vitellogenèse (V) sont au même stade. Aucun des jeunes ovocytes arrivés en fin de prévitellogenèse $(\mathrm{P} V)$ n'entre en vitellogenèse. La zone germinative $(\mathrm{G})$ bien que refoulée au centre de la gonade reste accolée à la paroi.

\section{FIG, 7}

Femelle de Lysmata seticaudata privée de ses pédoncules oculaires en hiver. Coupe d'un ovaire montrant que la vitellogenèse s'est déclenchée. Comme chez la femelle normale en reproduction (Fig. 6) aucun des jeunes ovocytes n'entre en vitellogenèse même s'il est en fin de prévitellogenèse. La zone germinative est visible à droite de la figure.

(Charniaux-Cotton et Touir, 1973) 


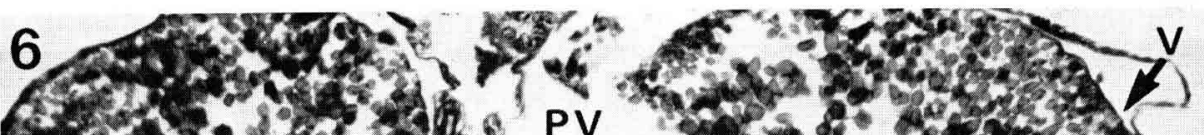

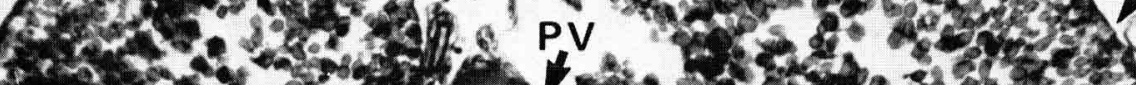

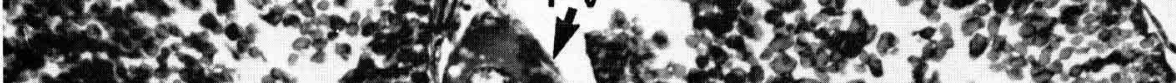
2.

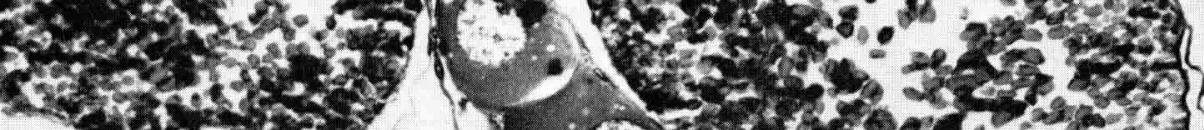

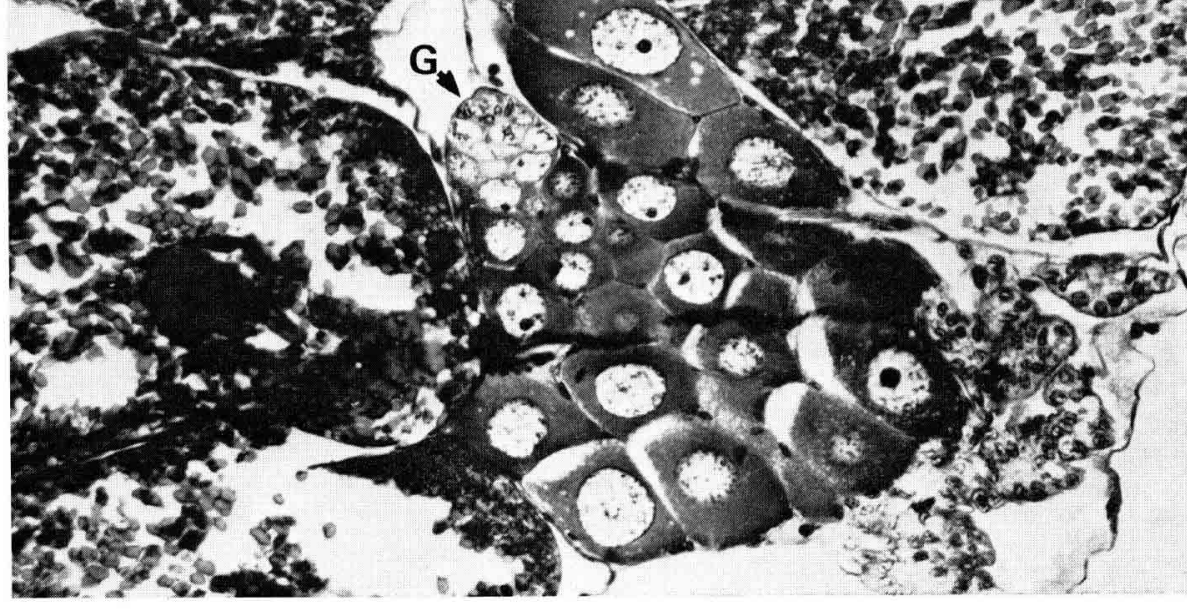

7 -

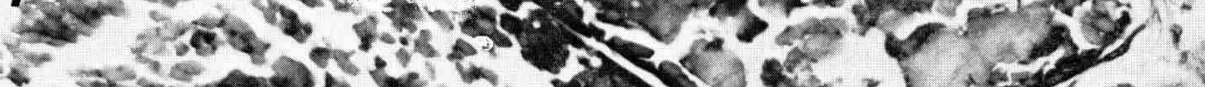

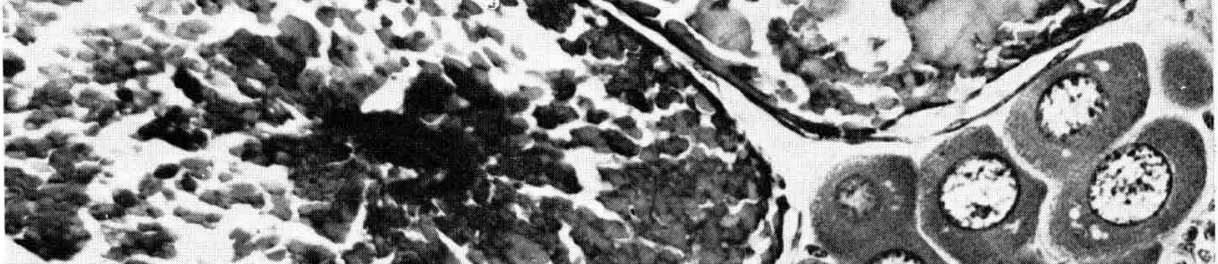

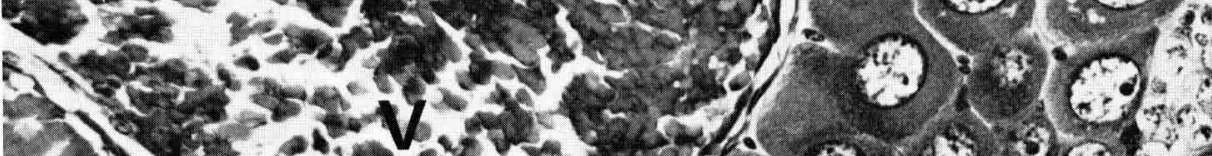

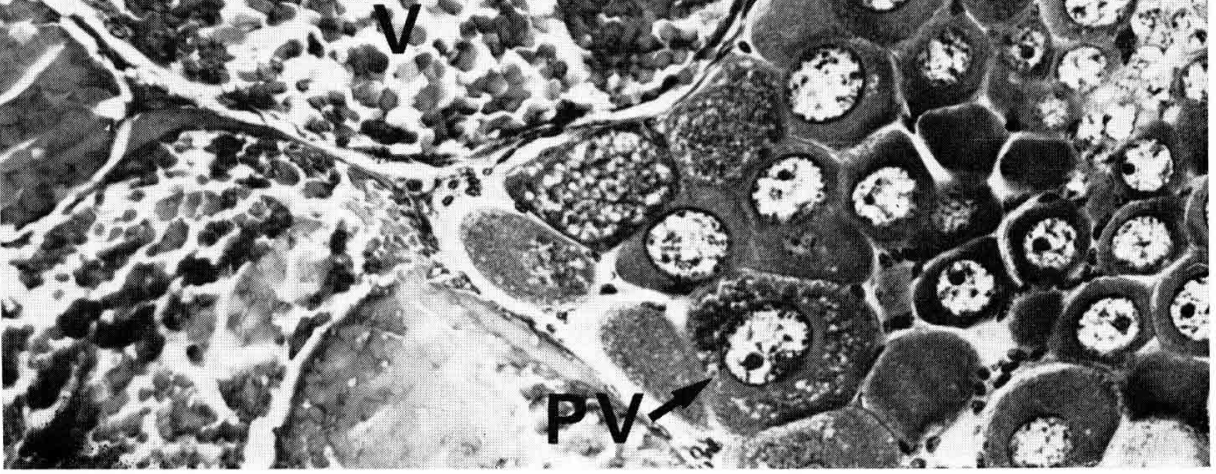

